\title{
Case Report of a Patient with Breast Metastasis from Gastric Cancer Treated with Paclitaxel and Ramucirumab Plus Regional Hyperthermia
}

\author{
JESSICA LUCCHETTI ${ }^{1}$, VINCENZO FORMICA ${ }^{1}$, GRETA GIULIANO ${ }^{1}$, \\ DEBORAH COLETTA ${ }^{1}$, LETIZIA GARGANO $^{1}$, DANIELE MOROSETTI ${ }^{2}$, \\ PIERPAOLO SILERI ${ }^{3}$, GIAMPIERO PALMIERI ${ }^{4}$ and MARIO ROSELLI ${ }^{1}$ \\ ${ }^{1}$ Medical Oncology Unit, ${ }^{2}$ Diagnostic Imaging Department, ${ }^{3}$ Surgery Department and \\ ${ }^{4}$ Anatomic Pathology Department, Tor Vergata University, Rome, Italy
}

\begin{abstract}
Background: Breast metastases from extra mammary tumors are extremely rare. Case Report: Here we report the case of a 50-year-old female with histologicallyconfirmed gastric cancer metastasis to the breast who was adequately treated with loco-regional hyperthermia plus standard second-line chemotherapy (paclitaxel plus ramucirumab). The best response achieved was a relatively long disease stabilization. Conclusion: Chemotherapy plus regional hyperthermia has been shown to have a synergistic antitumor effect and possible favorable immunomodulatory effects. Such an approach merits further investigation especially for the treatment of rare superficial metastatic sites.
\end{abstract}

Metastases to the breast from extra mammary neoplasms are extremely rare. At autoptic examination the incidence ranges between 1.7 and $6.6 \%$ while in studies with cytological samples the occurrence is reported to be $2.7 \%$ (1). So far, about 500 cases of secondary localizations to the breast from extra mammary tumors have been described in the scientific literature, with melanoma and lymphomas being the commonest primary malignancies associated with this clinical condition (1).

Owing to their rarity, no standard treatment has been defined for secondary breast tumors, and systemic chemotherapy or biological therapies are usually offered based on the primary tumor histology. Success of local treatment such as external-beam radiotherapy is also underreported and no data are available on local therapies other than radiotherapy.

Correspondence to: Jessica Lucchetti, Medical Oncology Unit, Tor Vergata University, Viale Oxford 81, 00133 Rome, Italy. E-mail: jess.lucchetti@gmail.com

Key Words: Breast, metastasis, gastric cancer, regional hyperthermia, case report.
Breast metastases from gastric adenocarcinoma have rarely been reported as single case reports.

We report a single case of breast metastasis from gastric tumor treated with regional hyperthermia (RHT) in combination with standard chemotherapy as per reported literature (2) for the treatment of soft tissue sarcomas or for tumors at superficial sites (such as skin, cutaneous neoplasm, breast or others).

\section{Case Description}

A 50-year-old female came to our observation in December 2016 with a diagnosis of metastatic gastric cancer. In her clinical history the following data were recorded: current smoker status, past history of arterial hypertension not currently on pharmacological treatment, removal of sebaceous cysts of the scalp, fibrocystic mastopathy treated in the past with estrogenic therapy for about 10 years.

Until December 2016 she had been treated for her cancer in another Institution. In May 2016, for abdominal pain in the upper quadrants associated with weight loss, the patient entered the Emergency Room. During the hospitalization, an upper GI endoscopy was performed that resulted in negative macroscopic alterations, however random biopsies at the body of the stomach were taken. Histological examination described chronic superficial gastritis and no evidence of cancer.

A following thorax-abdomen-pelvis CT scan demonstrated moderate abdominal ascites and thickening of the gastric wall, in particular at the level of the gastric greater curvature. Also, a lower GI endoscopy was negative for macroscopic lesions.

In June 2016, the patient underwent a diagnostic laparoscopy that revealed two peritoneal metastases on the surface of the greater curvature of the stomach. The bioptic analysis of the peritoneal implants revealed CK7+ CK20+ adenocarcinoma cells. 
A F-labeled fluoro-2-deoxyglucose Positron Emission Tomography - Computed Tomography (FDG PET-CT) scan in July 2016 was negative for neoplastic lesions, while a repeat upper GI endoscopy associated with endoscopic ultrasound examination in August 2016 revealed a rigid plaque with subverted mucosa and submucosa determining lumen substenosis at the gastric greater curvature. Biopsies were taken and adenocarcinoma with signet-ring cells, $\mathrm{CK} 7^{+}$, $\mathrm{CK} 20^{+}, \mathrm{Her}^{-}$and $\mathrm{CDX}_{2}^{-}$were demonstrated at microscopic examination. In the same period, a paracentesis was performed with cytological examination of the ascitic fluid, which described the presence of atypical epithelial cells suggestive of adenocarcinoma.

In September 2016, a first-line chemotherapy was started according to the EOX regimen (oxaliplatin, epirubicin, capecitabine).

Between September 2016 and November 2016 six cycles of EOX were administered. In November 2016 the patient noticed the growth of a well-defined, centimetric hard hyperchromic nodule in the left breast. Mammograms documented a single lesion at the superior-external left quadrant to which an excisional biopsy procedure was performed. Microscopic examination demonstrated infiltration of adenocarcinoma with signet- ring cells, estrogen receptor negative, HER2 negative, compatible with gastric origin.

Figure 1 shows the appearance of the left breast after the excisional biopsy procedure. Shortly after the breast biopsy (one month later), a re-growth of neoplastic lesions was noticed on the area surrounding the breast scar and a repeat CT scan also revealed peritoneal disease progression (Figure 2).

In December 2016, the patient was referred at our Institution for a second-line treatment and in the absence of a standard of care for breast metastasis from gastric cancer the combination of paclitaxel and ramucirumab plus regional hyperthermia (RHT) was proposed. The patient signed the informed consent.

Local hyperthermia was performed as per standard procedure by applying external heating plates at an energy of 200 watts, for approximately $45 \mathrm{~min}$, twice a week as well as before chemotherapy infusion. The standard paclitaxel plus ramucirumab schedule was administered: ramucirumab $8 \mathrm{mg} / \mathrm{kg}$ on days 1 and 15 , plus paclitaxel $80 \mathrm{mg} / \mathrm{m}^{2}$ intravenously on days 1,8 , and 15 of a 28 -day cycle.

After two months of therapy, a clinical benefit was reported by the patient, with less discomfort and soreness in the breast area and reduced local inflammation at the level of the breast gland. At physical examination, breast lesions appeared to be stable and a repeat CT scan also demonstrated improved left breast disease and stable disease in the peritoneum (Figure 3).

The patient remained on treatment for further three months, with accompanied stable radiologic disease and unchanged appearance at the left breast examination.

After approximately six months of therapy (last cycle in May 2017) a rapid radiologically-confirmed peritoneal progression occurred with performance status deterioration. Death occurred after nine months since the start of RHT plus bio-chemotherapy (August 2016).

\section{Discussion}

The first case of a breast secondary lesion from gastric cancer was reported in 1999 by an Italian group (3), however, breast metastasis from advanced gastric cancer (AGC) are more frequent among Orientals.

Approximately 40 cases of this rare presentation have, so far, been reported in the scientific literature. They occur at a relatively young age (around 45 years) (4), and the majority of them present histological features of signet-ring cell carcinoma, a histological subtype that occurs in approximately $10 \%$ of gastric cancers.

In $25-40 \%$ of the cases, breast metastasis is the only manifestation at the onset of the disease (4). Clinically, metastatic breast lesions present as non-tender, well-defined mobile nodules on physical examination (3). The most common localization is the upper outer quadrant of the left breast; bilateral involvement is present in $25 \%$ of the cases, and concomitant axillary lymph node infiltration is demonstrated in $15 \%$ of cases. Multiple metastatic breast nodules, or inflammatory breast carcinoma-like presentation are extremely rare (4).

Biopsy should always be performed. Immunohistochemistry of breast metastasis from AGC usually shows positivity for CEA and cytokeratin 7 and 20 and negativity for estrogen receptor (ER), progesterone receptor (PR) and C-erb-B2. GCDFP-15 (gross cystic disease fluid protein-15) is also absent (4).

Patients with breast metastasis from AGC have a poor prognosis as the median survival has been reported to be approximately 6 months since the diagnosis of metastasis. On that regard, our case compares favorably with the literature data as overall survival was as long as ten months since the occurrence of metastasis to the breast $(5,6)$.

As far as the pathogenesis is concerned, many hypotheses have been put forth to explain the occurrence of this rare condition. Amongst them the most trusted ones are: 1) increased blood supply to the breast in pre-menopausal women; 2) the presence of hormonal factors implicated in an aggressive biological behavior in young patients that induces metastasis to the breast (4).

In such cases, standard chemotherapy including docetaxel, cisplatin/oxaliplatin, S100/fluorouracil, is administered $(5,6)$.

More recently, a new biologic agent, the anti-vascular endothelial growth factor receptor (VEGFR) fully human monoclonal antibody ( $\operatorname{IgG} 1$ ) ramucirumab, has been approved for the treatment of advanced gastric cancer (AGC) (7). Ramucirumab is licensed as a single agent or in combination with paclitaxel for the treatment of AGC or 


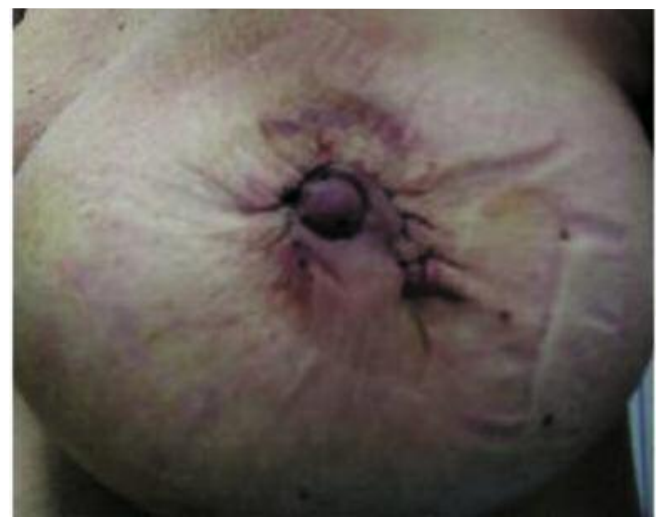

Figure 1. Appearance of the left breast after the excisional biopsy procedure.

gastro-esophageal junction adenocarcinoma if the disease has progressed despite the administration of fluoropyrimidine- or platinum-containing chemotherapy (7). No specific data are available on the activity of ramucirumab on secondary breast metastases from AGC.

In the patient described here, we choose to offer a multimodality therapeutic approach (chemotherapy plus RHT) as the best treatment opportunity for such an aggressive disease.

Heat exposure $\left(40^{\circ} \mathrm{C}-43^{\circ} \mathrm{C}\right)$ of cancer cells in preclinical studies have shown synergistic activity with ionizing radiation and chemotherapy (8) and on this basis a number of clinical trials have been conducted to investigate the safety and efficacy of regional hyperthermia (RHT) in combination with standard anti-cancer therapies.

Among them, the EORTC 62961-ESHO 95 Randomized Clinical Trial has recently demonstrated improved survival with RHT plus neoadjuvant doxorubicin, ifosfamide, and etoposide chemotherapy in patients with high risk localized soft tissue sarcomas (mainly liposarcoma, leiomyosarcoma or synovial sarcoma) as compared to chemotherapy alone. Apart from sarcomas, other types of solid tumors, such as germ cell tumors and bladder cancer, might derive benefit from the addition of RHT (2).

Currently, no data are available on the use of biological agents plus chemotherapy and RHT in breast metastasis from extramammary solid tumors. Here, we present a rare case of a woman with breast metastasis from AGC, treated with paclitaxel and ramucirumab in association with RHT after failure of standard first-line oxaliplatin/fluoropyrimidinebased therapy.

Ramucirumab plus paclitaxel has been demonstrated to be superior to paclitaxel monotherapy in a large randomized phase III trial (the RAINBOW study) and has become a standard of care as second line treatment for AGC since then (7).

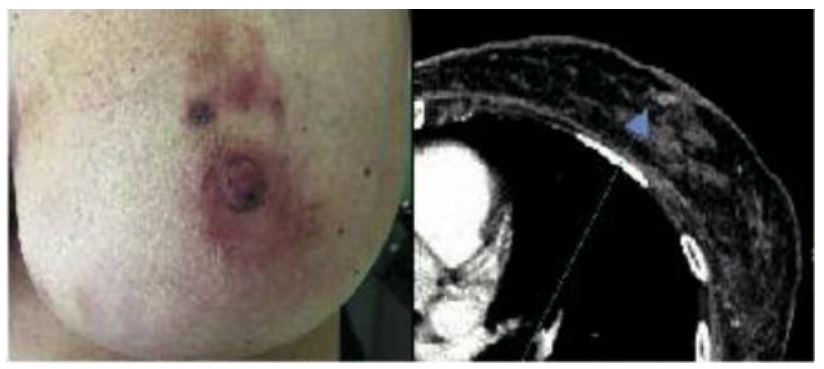

Figure 2. Progression of breast metastasis from gastric cancer before the second-line treatment with regional hyperthermia plus paclitaxel/ramusirumab. On the right panel, the respective CT scan shows a contrast-enhanced breast nodule (blue arrow).

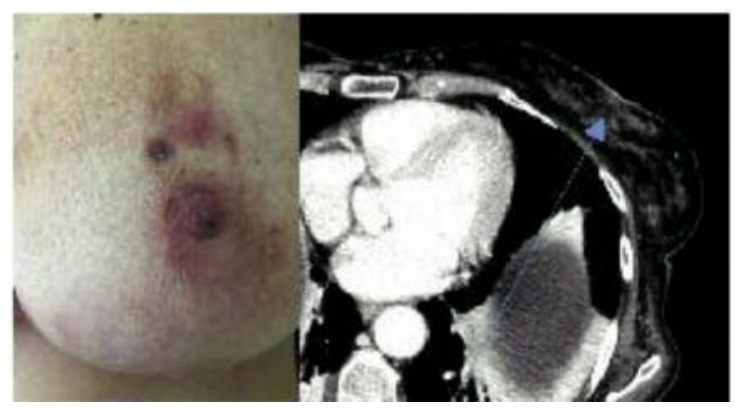

Figure 3. Appearance of the left breast after two months of second-line therapy with regional hyperthermia plus pacliaxel/ramusirumab. Clinically the breast lessions appeared to be stable. On the respective $C T$ scan the previously detected contrast-enhanced nodule was no longer visibly associated with normal glandural breast tissue and regular skin thickness (blue arrow).

RHT is available at our Institution (Tor Vergata University Hospital, Rome, Italy) in combination with standard chemotherapy as per reported literature for the treatment of soft tissue sarcomas or for tumors at superficial sites (such as skin, cutaneous neoplasm, breast or others) for which no standard treatment is recognized. Hyperthermia may have a number of biological and immunological anti-tumor modes of action.

Besides being a potential radio- and chemosensitizer, hyperthermia might generate very favorable immune signals and functions. It seems to work like an in situ vaccine. Its mechanism is based on the action of some danger signals such as heat shock proteins (like HSP70) and other molecules which are released by the heated tumor cells. HSP70 carries tumor peptides to dendritic cells (DCs) by binding to HSP receptors. DCs then process tumor peptides 
and cross-present tumor antigens via MHC class I molecules to $\mathrm{CD}^{+} \mathrm{T}$ cells (cytotoxic $\mathrm{T}$ cells, CTLs) with appropriate co-stimulation. Additionally, other danger signals might directly activate natural killer (NK) cells as part of the innate immune system. As a result of the above described molecular cascade, a tumor-specific immune response might be generated (9).

\section{Conclusion}

A phase III randomized trial has demonstrated that the addition of RHT to standard chemotherapy may improve patient outcome in specific disease settings such as soft tissue sarcomas (2). Its use in other rare oncological conditions, such as breast metastases from extramammary primary tumors still needs to be validated prospectively, however we present a case that lends support to investigate such an approach in rare oncological scenarios such as metastatic spread to superficial sites from visceral solid tumors.

\section{References}

1 Vizcaino I, Torregrosa A, Higueras V, Morote V, Cremades A, Torres V, Olmos S and Molins C: Metastasis to the breast from extramammary malignancies: a report of four cases and a review of literature. Eur Radiol 11: 1659-1665, 2001.

2 Issels RD, Lindner LH, Verweij J, Wessalowski R, Reichardt P, Wust P, Ghadjar P, Hohenberger P, Angele M, Salat C, Vujaskovic Z, Daugaard S, Mella O, Mansmann U, Dürr HR, Knösel T, Abdel-Rahman S, Schmidt M, Hiddemann W, Jauch $\mathrm{KW}$, Belka C and Gronchi A; European Organization for the Research and Treatment of Cancer-Soft Tissue and Bone Sarcoma Group and the European Society for Hyperthermic Oncology. Effect of neoadjuvant chemotherapy plus regional hyperthermia on long-term outcomes among patients with localized high-risk soft tissue sarcoma: The EORTC 62961-ESHO 95 Randomized Clinical Trial. JAMA Oncol 4: 483-492, 2018.
3 Di Cosimo S, Ferretti G, Fazio N, Mandalà M, Curigliano G, Bosari S, Intra M, Latronico A and Goldhirsch A: Breast and ovarian metastatic localization of signet cell gastric carcinoma. Ann Oncol 14: 803-804, 2003.

4 Boutis AL, Andreadis C, Patakiouta F and Mouratidou D:Gastric signet-ring adenocarcinoma presenting with breast metastasis. World J Gastroenterol 12: 2958-2961, 2006.

$5 \mathrm{He}$ CL, Chen P, Xia BL, Xiao Q and Cai FL: Breast metastasis of gastric signet-ring cell carcinoma: a case report and literature review. World J Surg Oncol 13: 120, 2015.

6 Iesato A, Oba T, Ono M, Hanamura T, Watanabe T, Ito T, Kanai T, Maeno K, Ishizaka K, Kitabatake H, Takeuchi D, Suzuki A, Nakayama $\mathbf{J}$ and Ito $\mathrm{K}$ : Breast metastases of gastric signet-ring cell carcinoma: a report of two cases and review of the literature. Onco Targets Ther 8: 91-97, 2014.

7 Elimova E, Lin Q, Song S and Ajani JA: Current status of ramucirumab in gastroesophageal adenocarcinoma. Future Oncol 13: 1585-1592, 2017.

8 Datta NR, Ordóñez SG, Gaipl US, Paulides MM, Crezee H, Gellermann J, Marder D, Puric E and Bodis S: Local hyperthermia combined with radiotherapy and-/or chemotherapy: Recent advances and promises for the future. Cancer Treat Rev 41: 742-753, 2015.

9 Chen T, Guo J, Han C, Yang M and Cao X: Heat Shock Protein 70 , released from heat-stressed tumor cells, initiates antitumor immunity by inducing tumor cell chemokine production and activating dendritic cells via TLR4 pathway. J Immunol 182: 1449-1459, 2009.
Received October 7, 2018

Revised October 21, 2018

Accepted October 22, 2018 